\title{
THE METRIC THEORY OF MIXED TYPE LINEAR FORMS
}

\author{
DETTA DICKINSON \\ Department of Mathematics \& Statistics \\ NUI Maynooth, Co. Kildare, Republic of Ireland \\ ddickinson@maths.nuim.ie \\ MUMTAZ HUSSAIN \\ Department of Mathematics and Statistics \\ Latrobe University, Melbourne, 3086 VIC, Australia \\ m.hussain@latrobe.edu.au
}

Received 20 October 2011

Accepted 27 June 2012

Published 6 November 2012

\begin{abstract}
In this paper the metric theory of Diophantine approximation of linear forms that are of mixed type is investigated. Khintchine-Groshev theorems are established together with the Hausdorff measure generalizations. The latter includes the original dimension results obtained in [H. Dickinson, The Hausdorff dimension of sets arising in metric Diophantine approximation, Acta Arith. 68(2) (1994) 133-140] as special cases.
\end{abstract}

Keywords: Diophantine approximation; Khintchine type theorems; system linear forms; Hausdorff measure; Hausdorff dimension.

Mathematics Subject Classification 2010: 11J83, 11J13, 11K60

\section{Introduction}

Let $\psi: \mathbb{R}^{+} \rightarrow \mathbb{R}^{+}$be a positive decreasing function such that $\psi(r) \rightarrow 0$ as $r \rightarrow$ $\infty$. Such a function will be referred to as an approximating function. Here and throughout, the system $q_{1} x_{1 i}+\cdots+\dot{q}_{m} x_{m i}$ of $n$ linear forms in $m$ variables will be written more concisely as $\mathbf{q} X$ and $|\mathbf{q}|$ denotes the supremum norm of the integer vector q. Let $W(m, n ; \psi)$ be the set of $X \in I^{m n}:=\left[-\frac{1}{2}, \frac{1}{2}\right]^{m n}$ such that the system of inequalities $\|\mathbf{q} X\|:=|\mathbf{q} X-\mathbf{p}|<\psi(|\mathbf{q}|)$ holds for infinitely many $(\mathbf{p}, \mathbf{q}) \in \mathbb{Z}^{n} \times$ $\left(\mathbb{Z}^{m} \backslash\{\mathbf{0}\}\right)$. The metric theory is well established for this set, see $[3,5,6,16,20]$ for details. The metric theory for the subset

$$
W_{0}(m, n ; \psi):=\left\{X \in I^{m n}:|\mathbf{q} X|<\psi(|\mathbf{q}|) \text { for i.m. } \mathbf{q} \in \mathbb{Z}^{m} \backslash\{\mathbf{0}\}\right\},
$$

where "i.m." means "infinitely many", of $W(m, n ; \psi)$ is established in [17] (the dimension of this set was obtained in [9]). The purpose of this note is to consider a 
set in which the forms of approximation are mixed. More precisely, let $W_{u}(m, n ; \psi)$ be the set of points $X \in I^{m n}$ such that

$$
\max \left(\left|\mathbf{q} \cdot \mathbf{x}^{(1)}-p_{1}\right|, \ldots,\left|\mathbf{q} \cdot \mathbf{x}^{(u)}-p_{u}\right|,\left|\mathbf{q} \cdot \mathbf{x}^{(u+1)}\right|, \ldots,\left|\mathbf{q} \cdot \mathbf{x}^{(n)}\right|\right)<\psi(|\mathbf{q}|)
$$

for infinitely many integer vectors $\mathbf{q} \in \mathbb{Z}^{m} \backslash\{0\}$ and $\mathbf{p} \in \mathbb{Z}^{u}$. Here, $\mathbf{x}^{(1)}, \ldots, \mathbf{x}^{(n)}$ are the column vectors of $X$. In this article we will obtain a complete Khintchine-Groshev theorem for $W_{u}(m, n ; \psi)$ together with the Hausdorff measure analogues.

The case $u=0, m=1$ will be omitted as $W_{0}(1, n ; \psi)=\{0\}$. It can be readily verified that $W_{u}(1, n ; \psi)=W(1, u ; \psi) \times\{0\}^{n-u}$ for $u<n$ so that

$$
\operatorname{dim} W_{u}(1, n ; \psi)=\operatorname{dim} W(1, u ; \psi) .
$$

If $\psi(r)=r^{-\tau}$, for some $\tau>0$, then $W_{u}(m, n ; \psi)$ will be denoted by $W_{u}(m, n ; \tau)$. This set was first considered in [12] when the Hausdorff dimension of $W_{1}(m, 2 ; \tau)$ for $m>1$, was obtained. More generally, the set $W_{u}(m, 2 ; \tau)$ is related to an exceptional set associated with the linearization of germs of complex analytic diffeomorphisms of $\mathbb{C}^{m}$ near a fixed point and is the $m$-dimensional version of Schröder's functional equation, see $[1,12,13]$ for further details.

In [10], the Hausdorff dimension of the set $W_{A}(m, n ; \tau)$, where $A$ is a subgroup of $\mathbb{Z}^{n}$ of dimension $u(\mathbf{p} \in A)$, was calculated and the following theorem was proved.

Theorem 1. When $m, n>0$ are integers,

$$
\operatorname{dim} W_{A}(m, n ; \tau)= \begin{cases}0 & \text { if } u=0, m=1, \\ (m-1) n+\frac{m+u}{\tau+1} & \text { if } \tau \geq \frac{m+u}{n}-1 .\end{cases}
$$

This theorem does not include the case $0<\tau \leq \frac{m+u}{n}-1$ and in fact includes a mistake. The result above does not hold for the cases $m+u \leq n$ if $\tau \cdot<\frac{m+u}{m+u-1}-1$. The mistake is corrected in the results of this paper and the remaining case is also dealt with.

To simplify notation the Vinogradov symbols « and $\gg$ will be used to indicate an inequality with an unspecified positive multiplicative constant depending only on $m, n$ and $u$. If $a \ll b$ and $a \gg b$ we write $a \asymp b$, and say that the quantities $a$ and $b$ are comparable. The $k$-dimensional Lebesgue measure of a set $A$ will be denoted by $|A|_{k}$. A dimension function is a function $f: \mathbb{R}^{+} \rightarrow \mathbb{R}^{+}$such that $f(r) \rightarrow 0$ as $r \rightarrow 0$ and such that it is increasing for $r \in\left(0, r_{0}\right)$ for some fixed $r_{0}$. Throughout the paper, $\mathcal{H}^{f}$ denotes the $f$-dimensional Hausdorff measure which will be fully defined in Sec. 3.1. Finally, for convenience, for a given approximating function $\psi$, define the function

$$
\Psi(r):=\frac{\psi(r)}{r}
$$




\section{Statements}

There are two cases and each subsection contains results about the Hausdorff measure, the Lebesgue measure and the Hausdorff dimension for that case.

\subsection{The case $m+u>n$}

The following theorem provides the complete measure theoretic description of $W_{u}(m, n ; \psi)$ when $m+u>n$.

Theorem 2. Let $m+u>n$ and $\psi$ be an approximating function. Let $f$ be a dimension function such that $r^{-m n} f(r)$ is non-increasing and $r^{-(m-1) n} f(r)$ is increasing. Then,

$$
\mathcal{H}^{f}\left(W_{u}(m, n ; \psi)\right)= \begin{cases}0 & \text { if } \sum_{r=1}^{\infty} f(\Psi(r)) \Psi(r)^{-(m-1) n} r^{m+u-1}<\infty, \\ \mathcal{H}^{f}\left(I^{m n}\right) & \text { if } \sum_{r=1}^{\infty} f(\Psi(r)) \Psi(r)^{-(m-1) n} r^{m+u-1}=\infty .\end{cases}
$$

The requirement that $r^{-m n} f(r)$ be non-increasing is a natural and not particularly restrictive condition. If $f$ is such that $r^{-m n} f(r) \rightarrow \infty$ as $r \rightarrow 0$ then $\mathcal{H}^{f}\left(I^{m n}\right)=\infty$ and if $f(r)=r^{m n}$ then the Hausdorff measure $\mathcal{H}^{f}$ is proportional to $m n$-dimensional Lebesgue measure and the result is the natural analogue of the Khintchine-Groshev theorem for $W_{u}(m, n ; \psi)$. Indeed if $u=n$ then these wellknown theorems $[15,19]$ are obtained together with the classical results of Jarník originally proved in [18] for $m=1$ and [11] for $m>1$. These theorems were put in a more general context in [3]; also in that paper some of the conditions on the dimension and approximating functions (used in $[11,15,18,19])$ were shown to be unnecessary. When $u=0$ Theorem 2 reduces to [17, Theorem 1].

Corollary 1. Let $m+u>n$ and $\psi$ be an approximating function. Then

$$
\left|W_{u}(m, n ; \psi)\right|_{m n}= \begin{cases}0 & \text { if } \sum_{r=1}^{\infty} \Psi(r)^{n} r^{m+u-1}<\infty \\ 1 & \text { if } \sum_{r=1}^{\infty} \Psi(r)^{n} r^{m+u-1}=\infty\end{cases}
$$

From this, it can easily be seen that for $\tau>\frac{m+u}{n}-1, W_{u}(m, n ; \tau)$ is a null set. The following corollary gives the Hausdorff measure and dimension when $f(r)=r^{s}$, $s>0$. We refer the reader to $[2,11]$ for more details. For convenience in both this corollary and Corollary 5 the notation

$$
T=\inf \left\{s: \sum_{r=1}^{\infty} \Psi(r)^{s-(m-1) n} r^{m+u-1}<\infty\right\}
$$

will be used. 
Corollary 2. Let $m+u>n$ and $\psi$ be an approximating function. Let $s$ be such that $(m-1) n<s \leq m n$. Then,

$$
\mathcal{H}^{s}\left(W_{u}(m, n ; \psi)\right)= \begin{cases}0 & \text { if } \sum_{r=1}^{\infty} \Psi(r)^{s-(m-1) n} r^{m+u-1}<\infty, \\ \mathcal{H}^{s}\left(I^{m n}\right) & \text { if } \sum_{r=1}^{\infty} \Psi(r)^{s-(m-1) n} r^{m+u-1}=\infty .\end{cases}
$$

Also, $\operatorname{dim} W_{u}(m, n ; \psi)=\min \{T, m n\}$.

Proof. All but the last line follows directly from Theorem 2 . For the last line note that if $\psi$ is such that $T>m n$ then $W_{u}\left(m, n ; \frac{m+u}{n}-1\right) \subset W_{u}(m, n ; \psi)$. The result then follows from the preceding corollary.

Finally, for completeness, the dimension result for $W_{u}(m, n ; \tau)$ is given for $m+$ $u>n$. This follows directly from the two preceding corollaries.

Corollary 3. For $m+u>n$,

$$
\operatorname{dim} W_{u}(m, n ; \tau)= \begin{cases}(m-1) n+\frac{m+u}{\tau+1} & \text { if } \tau>\frac{m+u}{n}-1, \\ m n & \text { if } \tau \leq \frac{m+u}{n}-1 .\end{cases}
$$

Together, these results contain the results from [10] and also give the Hausdorff measure at the critical exponent.

\subsection{The case $m+u \leq n$}

From now on let $d=(m-1) n+m+u-1$. For $m+u \leq n$ the conditions on the dimension function in Theorem 2 change. This change is due to the fact that if $X \in W_{u}(m, n ; \psi)$ and $m+u \leq n$ then $X$ is over-determined and lies in a subset of strictly lower dimension than $m n$. More notation is needed. For each $m \times n$ matrix $X \in \mathbb{R}^{m n}$ with column vectors $\mathbf{x}^{(1)}, \ldots, \mathbf{x}^{(n)}$ define $\tilde{X}$ to be the $m \times(n-u)$ matrix with column vectors $\mathbf{x}^{(u+1)}, \ldots, \mathbf{x}^{(n)}$. The set $\Gamma \subset \mathbb{R}^{m n}$ is the set of $X \in \mathbb{R}^{m n}$ such that the determinant of each $m \times m$ minor of $\tilde{X}$ is zero. It will now be proved that $W_{u}(m, n ; \psi) \subset \Gamma$ when $m+u \leq n$.

Lemma 1. For $m+u \leq n$ the set $W_{u}(m, n ; \psi)$ is contained in $\Gamma$ and $\operatorname{dim} \Gamma=d<$ mn. Thus

$$
\operatorname{dim} W_{u}(m, n ; \psi) \leq d
$$

Proof. First the dimension of $\Gamma$ is obtained. Assume that $m=n$ (i.e. $u=0$ ). The dimension of the set of $X \in \mathbb{R}^{m^{2}}$ such that $\operatorname{det} X=0$ is $m^{2}-1=d$ as required. 
Now assume that $u>0$ and $m+u=n$. The number of variables in the first $u$ columns is $m u$. Each $X \in \Gamma$ satisfies $\operatorname{det} \tilde{X}=0$. As before, the dimension of the set of $\tilde{X} \in \mathbb{R}^{m^{2}}$ such that $\operatorname{det} \tilde{X}=0$ is $m^{2}-1$. Thus, the dimension of $\Gamma$ is $m^{2}-1+m u=d$.

Finally, for $m+u<n$ a similar argument is used. The first $u$ columns contain $m u$ variables. Each $m \times m$ minor of $\tilde{X}$ has determinant zero so $\tilde{X}$ has rank at most $m-1$. Take any $m-1$ columns which obviously contain $(m-1) m$ variables. Then, the set of $\tilde{X}$ such that each $m \times m$ minor, containing the chosen columns, has determinant zero has dimension $(m-1) m+(n-m-u+1)(m-1)$. Clearly, there are only $\left(\begin{array}{c}n-u \\ m-1\end{array}\right)$ choices for the $m-1$ columns. Thus, the dimension of $\Gamma$ is $(m-1) m+(n-m-u+1)(m-1)+m u=(m-1) n+m+u-1=d$ as required.

If a matrix $X \in \mathbb{R}^{m^{2}}$ satisfies $|\mathbf{q} X|<\psi(|\mathbf{q}|)$ for infinitely many vectors $\mathbf{q} \in$ $\mathbb{Z}^{m}$ then $\operatorname{det} X=0$. This can easily be seen by assuming the contrary; i.e. that $\operatorname{det} X=c \neq 0$. Then, solving the equations $\mathbf{q} \cdot \mathbf{x}^{(i)}=\varepsilon_{i}$ for $i=1, \ldots, n$ where $\varepsilon_{i}<\psi(|\mathbf{q}|)$ gives $|\mathbf{q}| \ll \psi(|\mathbf{q}|)$ which is clearly impossible for $|\mathbf{q}|$ sufficiently large. If $X \in W_{u}(m, n ; \psi)$ then $|\mathbf{q} \tilde{X}|<\psi(|\mathbf{q}|)$ for infinitely many $\mathbf{q} \in \mathbb{Z}^{m}$. Thus, if $m+u \leq n$, each $m \times m$ minor of $\tilde{X}$ has determinant zero and $X \in \Gamma$.

The main results for this section are now stated.

Theorem 3. Let $m+u \leq n$ and $\psi$ be an approximating function. Let $f$ and $r^{-(n-m-u+1)(m-1)} f(r)$ be dimension functions. Assume that $r^{-d} f(r)$ is nonincreasing and $r^{-(m-1) n} f(r)$ is increasing. Then

$$
\mathcal{H}^{f}\left(W_{u}(m, n ; \psi)\right)=0 \quad \text { if } \sum_{r=1}^{\infty} f(\Psi(r)) \Psi(r)^{-(m-1) n} r^{m+u-1}<\infty .
$$

On the other hand, if

$$
\sum_{r=1}^{\infty} f(\Psi(r)) \Psi(r)^{-(m-1) n} r^{m+u-1}=\infty,
$$

then

$$
\mathcal{H}^{f}\left(W_{u}(m, n ; \psi)\right)=\left\{\begin{array}{lll}
\infty & \text { if } r^{-d} f(r) \rightarrow \infty & \text { as } r \rightarrow 0, \\
K & \text { if } r^{-d} f(r) \rightarrow C & \text { as } r \rightarrow 0
\end{array}\right.
$$

for some constant $0 \leq C<\infty$. If $C>0$, then $0<K<\infty$ and if $C=0$, then $K=0$.

Note that if $f$ satisfies $r^{-d} f(r) \rightarrow C>0$ as $r \rightarrow 0$ then the Hausdorff measure $\mathcal{H}^{f}$ is comparable to $d$-dimensional Lebesgue measure. Also, if $f(r)=r^{d}$, then the following analogue of the Khintchine-Groshev theorem is obtained. 
Corollary 4. Let $m+u \leq n, \psi$ be an approximating function and assume that the conditions of Theorem 3 hold for the dimension function $f(r)=r^{d}$. Then

$$
\left|W_{u}(m, n ; \psi)\right|_{d}= \begin{cases}0 & \text { if } \sum_{r=1}^{\infty} \psi(r)^{m+u-1}<\infty, \\ K & \text { if } \sum_{r=1}^{\infty} \psi(r)^{m+u-1}=\infty,\end{cases}
$$

where $0<K<\infty$.

Similarly, the $m+u \leq n$ analogue of Corollary 2 holds; for the last line the same argument, with appropriate changes, as in Corollary 2 is used. For convenience the notation

$$
S=\sum_{r=1}^{\infty} \Psi(r)^{s-(m-1) n} r^{m+u-1}
$$

is used.

Corollary 5. Let $m+u \leq n$ and $\psi$ be an approximating function. Let $s$ be such that $(m-1) n<s \leq d$. Then,

$$
\mathcal{H}^{s}\left(W_{u}(m, n ; \psi)\right)= \begin{cases}0 & \text { if } S<\infty \\ \mathcal{H}^{s}(\Gamma) & \text { if } S=\infty \text { and } s<d \\ K & \text { if } S=\infty \text { and } s=d\end{cases}
$$

where $0<K<\infty$.

Also, $\operatorname{dim} W_{u}(m, n ; \psi)=\min \{T, d\} .($ Note that $T$ is defined in (1).)

The above corollary gives the Hausdorff dimension of $W_{u}(m, n ; \tau)$ when $m+u \leq$ $n$ and also the Hausdorff measure at the critical exponent. Finally, the analogue of Corollary 3 is given. This contains some of the results from [10] and corrects a mistake in that paper.

Corollary 6. For $m+u \leq n$,

$$
\operatorname{dim} W_{u}(m, n ; \tau)= \begin{cases}(m-1) n+\frac{m+u}{\tau+1}, & \text { if } \tau>\frac{m+u}{m+u-1}-1, \\ d & \text { if } \tau \leq \frac{m+u}{m+u-1}-1 .\end{cases}
$$

\section{Basic Definitions and Auxiliary Results}

In this section the definitions of some fundamental concepts are given together with some of the auxiliary results needed in the proofs of Theorems 2 and 3 . 


\subsection{Hausdorff measure and dimension}

Below is a brief introduction to Hausdorff $f$-measure and dimension. For further details see $[7,14]$. Let $F \subset \mathbb{R}^{n}$. For any $\rho>0$ a countable collection $\left\{B_{i}\right\}$ of balls in $\mathbb{R}^{n}$ with diameters $\operatorname{diam}\left(B_{i}\right) \leq \rho$ such that $F \subset \bigcup_{i} B_{i}$ is called a $\rho$-cover of $F$. Define

$$
\mathcal{H}_{\rho}^{f}(F)=\inf \sum_{i} f\left(\operatorname{diam}\left(B_{i}\right)\right)
$$

where the infimum is taken over all possible $\rho$-covers of $F$. The Hausdorff $f$-measure of $F$ is

$$
\mathcal{H}^{J}(F)=\lim _{\rho \rightarrow 0} \mathcal{H}_{\rho}^{f}(F)
$$

In the particular case when $f(r)=r^{s}$ with $s>0$, we write $\mathcal{H}^{s}$ for $\mathcal{H}^{f}$ and the measure is referred to as $s$-dimensional Hausdorff measure. The Hausdorff dimension of $F$ is denoted by $\operatorname{dim} F$ and is defined as

$$
\operatorname{dim} F:=\inf \left\{s \in \mathbb{R}^{+}: \mathcal{H}^{s}(F)=0\right\} .
$$

Before the structure of $W_{u}(m, n ; \psi)$ is described we introduce a function $\omega$ needed in the proof of Theorem 2 for technical reasons.

\subsection{The function $\omega$}

Let $\omega$ be a positive real increasing function such that $\omega(t) \rightarrow \infty$ as $t \rightarrow \infty$ and such that there exists $C>1$ so that for $t$ sufficiently large $\omega(2 t)<C \omega(t)$. This function is chosen in such a way that the sum

$$
\sum_{r=1}^{\infty} f(\Psi(r)) \Psi(r)^{-(m-1) n} r^{m+u-1} \omega(r)^{-n}
$$

will converge (respectively, diverge) if and only if the sum

$$
\sum_{r=1}^{\infty} f(\Psi(r)) \Psi(r)^{-(m-1) n} r^{m+u-1}
$$

converges (respectively, diverges). This is always possible; see [8] for the example below. Clearly if the sum in (2) diverges then so does the sum in (3). On the other hand, suppose that the sum in (3) diverges. Then there exists a strictly increasing sequence of positive integers $\left\{r_{i}\right\}_{i \in \mathbb{N}}$ such that

$$
\sum_{r_{i-1} \leq r \leq r_{i}}^{\infty} f(\Psi(r)) \Psi(r)^{-(m-1) n} r^{m+u-1}>1
$$

and $r_{i}>2 r_{i-1}$. Define $\omega$ to be the step function $\omega(r)=i^{\frac{1}{n}}$ for $r_{i-1} \leq r \leq r_{i}$ and $\omega$ satisfies the required properties. 


\subsection{The structure of $W_{u}(m, n ; \psi)$}

For $\mathbf{q} \in \mathbb{Z}^{m}, \mathbf{p}=\left(p_{1}, \ldots, p_{u}\right) \in \mathbb{Z}^{u}$ the resonant set $R_{\mathbf{p}, \mathbf{q}}$ is the set

$R_{\mathrm{p}, \mathrm{q}}$

$$
=\left\{X \in I^{m n}:\left|\mathbf{q} \cdot \mathbf{x}^{(1)}-p_{1}\right|=\cdots=\left|\mathbf{q} \cdot \mathbf{x}^{(u)}-p_{u}\right|=\left|\mathbf{q} \cdot \mathbf{x}^{(u+1)}\right|=\cdots=\left|\mathbf{q} \cdot \mathbf{x}^{(n)}\right|=0\right\} .
$$

These sets are hyperplanes of dimension $(m-1) n$ and are contained in $W_{u}(m, n ; \psi)$ for all functions $\psi$. Let $\Re=\left\{R_{\mathbf{p}, \mathbf{q}}: \mathbf{q} \in \mathbb{Z}^{m} \backslash\{\mathbf{0}\}, \mathbf{p} \in \mathbb{Z}^{u}\right\}$. Given an approximating function $\Psi$ and a resonant set $R_{\mathbf{p}, \mathbf{q}}$, define the $\Psi$-neighborhood of $R_{\mathbf{p}, \mathbf{q}}$ as

$$
\tilde{\Delta}\left(R_{\mathbf{p}, \mathbf{q}}, \Psi(|\mathbf{q}|)\right)=\left\{X \in I^{m n}: \operatorname{dist}\left(X, R_{\mathbf{p}, \mathbf{q}}\right) \leq \Psi(|\mathbf{q}|)\right\}
$$

where $\operatorname{dist}\left(X, R_{\mathbf{p}, \mathbf{q}}\right):=\inf \left\{|X-Y|: Y \in R_{\mathbf{p}, \mathbf{q}}\right\}$. Notice that if $m=1$ then the resonant sets are points and the sets $\tilde{\Delta}\left(R_{\mathbf{p}, \mathbf{q}}, \Psi(|\mathbf{q}|)\right)$ are balls centered at these points.

Let

$$
\Lambda(m, n ; \Psi)=\left\{X \in I^{m n}: X \in \tilde{\Delta}\left(R_{\mathbf{p}, \mathbf{q}}, \Psi(|\mathbf{q}|)\right) \text { for i.m. }(\mathbf{p}, \mathbf{q}) \in \mathbb{Z}^{u} \times\left(\mathbb{Z}^{m} \backslash\{\mathbf{0}\}\right)\right\}
$$

and for any $t \in \mathbb{N}$, define

$$
\Delta(\Psi, t):=\bigcup_{\mathbf{q} \in J(t)} \bigcup_{\substack{\mathbf{p} \in \mathbb{Z}^{\mathbf{z}} \\|\mathbf{p}| \leq|\mathbf{q}|}} \tilde{\Delta}\left(R_{\mathbf{p}, \mathbf{q}}, \Psi(|\mathbf{q}|)\right)
$$

where

$$
J(t)=\left\{\mathbf{q} \in \mathbb{Z}^{m} \backslash\{\mathbf{0}\}: \frac{2^{t}}{\omega\left(2^{t}\right)}<|\mathbf{q}|<2^{t}\right\} .
$$

Then, $\Lambda(m, n ; \Psi)$ can be written as a lim sup set so that

$$
\Lambda(m, n ; \Psi)=\bigcap_{N=1}^{\infty} \bigcup_{t=N}^{\infty} \Delta(\Psi, t) .
$$

It can be readily verified that

$$
\Lambda\left(m, n ; \frac{1}{m} \psi\right) \subset W_{u}(m, n ; \psi) \subset \Lambda(m, n ; \psi) .
$$

In the next lemma the convergence half of both theorems is proved. For convergence no conditions on the dimension function are needed and the proof is a straightforward covering and counting argument.

Lemma 2. Let $\psi$ be an approximating function and let $f$ be a dimension function. If

$$
\sum_{r=1}^{\infty} f(\Psi(r)) \Psi(r)^{-(m-1) n} r^{m+u-1}<\infty
$$


then

$$
\mathcal{H}^{f}\left(W_{u}(m, n ; \psi)\right)=0
$$

Proof. The natural cover of $W_{u}(m, n ; \psi)$ given by (4) and (5) is used. From this it follows that for each $Q \in \mathbb{N}$

$$
W_{u}(m, n ; \psi) \subset \bigcup_{r>Q} \bigcup_{|\mathbf{q}|=r} \bigcup_{\substack{\mathbf{p} \in \mathbf{Z}^{u} \\|\mathbf{p}|<|\mathbf{q}|}} \tilde{\Delta}\left(R_{\mathbf{p}, \mathbf{q}}, \Psi(|\mathbf{q}|)\right) .
$$

For each resonant set $R_{\mathbf{p}, \mathbf{q}}$, the set $\tilde{\Delta}\left(R_{\mathbf{p}, \mathbf{q}}, \Psi(|\mathbf{q}|)\right)$ can be covered by a collection of $m n$-dimensional closed hypercubes with disjoint interior and sidelength $\Psi(|\mathbf{q}|)$. It can be readily verified that the number $N$ of such hypercubes satisfies $N \ll$ $\Psi(|\mathbf{q}|)^{-(m-1) n}$. Thus,

$$
\begin{aligned}
\mathcal{H}^{f}\left(W_{u}(m, n ; \psi)\right) & \leq \sum_{r>Q} \sum_{|\mathbf{q}|=r} \sum_{\substack{\mathbf{p} \in \mathcal{Z}^{u} \\
|\mathbf{p}|<|\mathbf{q}|}} \Psi(|\mathbf{q}|)^{-(m-1) n} f(\Psi(|\mathbf{q}|)) \\
& \ll \sum_{r>Q} r^{m+u-1} f(\Psi(r)) \Psi(r)^{-(m-1) n} \rightarrow 0
\end{aligned}
$$

as $Q \rightarrow \infty$. Thus $\mathcal{H}^{f}\left(W_{u}(m, n ; \psi)\right)=0$, as required.

\section{Proof of Theorem 2: The Divergence Case}

\subsection{Ubiquitous systems}

For the divergence part of Theorem 2 the idea of a locally ubiquitous system is used. We present a simplified version of a more abstract framework developed in $[3,5]$ from the original definition in [12]. In our case the required measure and intersection conditions in [3] are trivially satisfied.

Definition 1. Let $\rho: \mathbb{R}^{+} \rightarrow \mathbb{R}^{+}$be a function such that $\rho(r) \rightarrow 0$ as $r \rightarrow \infty$. Let $B$ be an arbitrary ball with center in $I^{m n}$ and radius at most $r_{o}(m, n, u)$. Suppose there exist a function $\rho$ and an absolute constant $\kappa>0$ such that

$$
|B \cap \Delta(\rho, t)|_{m n} \geq \kappa|B|_{m n} \quad \text { for } t \geq t_{o}(B)
$$

where $\Delta(\rho, t)$ is defined in (4). Then $\Re$ is said to be a locally ubiquitous system relative to $\rho$.

Loosely speaking the definition of local ubiquity says that the set $\Delta(\rho, t)$ locally approximates the underlying space $I^{m n}$ in terms of the Lebesgue measure. The function $\rho$ will be referred to as the ubiquity function. The actual value of $\kappa$ in the above definition is irrelevant, only its existence is important. In practice local ubiquity is usually established using standard results such as Dirichlet's theorem or Minkowski's Convex Body Theorem, regarding the distribution of the resonant 
sets; from which the function $\rho$ arises naturally. Clearly if $|\Delta(\rho, t)|_{m n} \rightarrow 1$ as $t \rightarrow \infty$ then $\Re$ is locally ubiquitous.

The following theorem is a simplified version of [5, Theorem 1].

Theorem 4. Assume that there exists $0<\lambda<1$ such that the function $\rho$ satisfies $\rho\left(2^{t+1}\right)<\lambda \rho\left(2^{t}\right)$ for all $t \in \mathbb{N}$. Suppose that $\Re$ is locally ubiquitous relative to $\rho$ and $\psi$ is an approximating function. Let $f$ be a dimension function such that $r^{-m n} f(r)$ is non-increasing. Furthermore suppose that $r^{-(m-1) n} f(r)$ is increasing. Then

$$
\mathcal{H}^{f}\left(W_{u}(m, n ; \psi)\right)=\mathcal{H}^{f}\left(I^{m n}\right) \quad \text { if } \sum_{n=1}^{\infty} \frac{f\left(\Psi\left(2^{t}\right)\right) \Psi\left(2^{t}\right)^{-(m-1) n}}{\rho\left(2^{t}\right)^{n}}=\infty .
$$

To establish ubiquity we need two technical lemmas, the first of which is proved in [10] and is the analogue of Dirichlet's theorem.

Lemma 3. For all $t>t_{0}(m, n, u)$ and for each $X \in I^{m n}$ there exist $\mathbf{q} \in \mathbb{Z}^{m} \backslash\{0\}$ and $\mathbf{p} \in \mathbb{Z}^{u}$ with $|\mathbf{q}|,|\mathbf{p}| \leq 2^{t}$ such that

$$
\max \left\{\left|\mathbf{q} \cdot \mathbf{x}^{(i)}-p_{i}\right|,\left|\mathbf{q} \cdot \mathbf{x}^{(j)}\right|\right\}<(m+2) 2^{\frac{u}{n}}\left(2^{t}\right)^{-\frac{m+u}{n}+1}
$$

for $i=1, \ldots, u$ and $j=u+1, \ldots, n$.

In the above lemma the $\mathbf{p}$ which has been shown to exist obviously satisfies $|\mathbf{p}|<\frac{1}{2}|\mathbf{q}|+1$ as otherwise $|\mathbf{q} X-\mathbf{p}|>1$. The following lemma is a slight modification of a result from $[10]$. The only difference is that instead of $\log$ the function $\omega$ is used.

Lemma 4. The family $\Re$ is locally ubiquitous with respect to the function $\rho: \mathbb{N} \rightarrow$ $\mathbb{R}^{+}$where

$$
\rho(t)=(m+2) 2^{\frac{u}{n}}\left(2^{t}\right)^{-\frac{m+u}{n}} \omega(t) .
$$

The divergence of

$$
\sum_{t=1}^{\infty} f\left(\Psi\left(2^{t}\right)\right) \Psi\left(2^{t}\right)^{-(m-1) n}\left(2^{t}\right)^{m+u} \omega\left(2^{t}\right)^{-n}
$$

is not difficult to establish from the divergence of

$$
\sum_{r=1}^{\infty} f(\Psi(r)) \Psi(r)^{-(m-1) n} r^{m+u-1} \omega(r)^{-n}
$$

by using the same argument as in the proof of [3, Corollary 3] (similar to that of Cauchy's Condensation Test). The monotonicity conditions in the statement of the theorem and the condition on the ubiquity function are both needed.

This completes the proof of Theorem 2. 


\section{Proof of Theorem 3: The Divergence Case}

First, we consider in more detail the structure of $W_{u}(m, n ; \psi)$ by following an argument from [10]. For each $X \in W_{u}(m, n ; \psi)$ let $\tilde{X}=\left(\mathbf{x}^{(u+1)} \ldots \mathbf{x}^{(n)}\right)$. From the proof of Lemma 1 the rank of $\tilde{X}$ is at most $m-1$. Now, we restrict ourselves to those $X \in W_{u}(m, n ; \psi)$ for which $\tilde{X}$ has rank $m-1$ : It can be readily verified that the set of $X$ for which $\tilde{X}$ has lower rank is of strictly lower dimension. Define a subset $\widehat{W}_{u}(m, n ; \psi)$ of $W_{u}(m, n ; \psi)$ so that the column vectors $\mathbf{x}^{(u+1)}, \ldots, \mathbf{x}^{(m+u-1)}$ are linearly independent. Let $\Gamma^{\prime} \subset \Gamma$ be the set of points $X \in \Gamma$ such that $\tilde{X}$ has rank $m-1$ and $\mathbf{x}^{(u+1)}, \ldots, \mathbf{x}^{(m+u-1)}$ are the linearly independent vectors.

Let $G$ be the set of points of the form

$$
\left(\mathbf{x}^{(1)}, \ldots, \mathbf{x}^{(m+u-1)}, \sum_{j=1}^{m-1} a_{j}^{(m+u)} \mathbf{x}^{(u+j)}, \ldots, \sum_{j=1}^{m-1} a_{j}^{(n)} \mathbf{x}^{(u+j)}\right),
$$

where

$$
\left(\mathbf{x}^{(1)}, \ldots, \mathbf{x}^{(m+u-1)}\right) \in \widehat{W}_{u}(m, m+u-1 ; \psi)
$$

and $a_{j}^{(i)} \in\left(-\frac{1}{m-1}, \frac{1}{m-1}\right)$ for $m+u \leq i \leq n$. Note that

$$
\begin{aligned}
\left|\mathbf{q} \cdot \sum_{j=1}^{m-1} a_{j}^{(i)} \mathbf{x}^{(u+j)}\right| & =\left|\sum_{j=1}^{m-1} a_{j}^{(i)} \mathbf{q} \cdot \mathbf{x}^{(u+j)}\right| \leq \sum_{j=1}^{m-1}\left|a_{j}^{(i)}\right|\left|\mathbf{q} \cdot \mathbf{x}^{(u+j)}\right| \\
& \leq\left(\sum_{j=1}^{m-1}\left|a_{j}^{(i)}\right|\right) \psi(|\mathbf{q}|) \leq \psi(|\mathbf{q}|)
\end{aligned}
$$

for $m+u \leq i \leq n$. It follows that $G \subseteq W_{u}(m, n ; \psi)$.

Now define the function

$$
\eta: \widehat{W}_{u}(m, m+u-1 ; \psi) \times\left(-\frac{1}{m-1}, \frac{1}{m-1}\right)^{(n-m-u+1)(m-1)} \rightarrow G
$$

by

$$
\begin{array}{r}
\eta\left(\mathbf{x}^{(1)}, \ldots, \mathbf{x}^{(m+u-1)}, a_{1}^{m+u}, \ldots, a_{m+u-1}^{m+u}, \ldots, a_{1}^{n-m-u+1}, \ldots, a_{m+u-1}^{n-m-u+1}\right) \\
\quad=\left(\mathbf{x}^{(1)}, \ldots, \mathbf{x}^{m+u-1}, \sum_{j=1}^{m+u-1} a_{j}^{(m+u)} \mathbf{x}^{(j)}, \ldots, \sum_{j=1}^{m+u-1} a_{j}^{n-m-u+1} \mathbf{x}^{(j)}\right) .
\end{array}
$$

It is shown in [10] that $\eta$ is a bijection and satisfies a bi-Lipschitz condition.

The following lemma follows directly from [14, Corollary 2.4].

Lemma 5. Let $f$ be a dimension function. Suppose that $L \subset \mathbb{R}^{l}, M \subset \mathbb{R}^{k}$ and $\eta$ : $L \rightarrow M$ is a bijection satisfying a bi-Lipschitz condition. Then $\mathcal{H}^{f}(L) \asymp \mathcal{H}^{f}(\eta(L))$. Thus $\eta$ preserves a zero-full law. 
To prove divergence the technique of "slicing" introduced in [4] will be used. It is first necessary to introduce a little notation. Suppose that $V$ is a linear subspace of $\mathbb{R}^{k}, V^{\perp}$ will be used to denote the linear subspace of $\mathbb{R}^{k}$ orthogonal to $V$. Further $V+a:=\{v+a: v \in V\}$ for $a \in V^{\perp}$. The following lemma was proved in [4].

Lemma 6. Let $l, k \in \mathbb{N}$ be such that $l \leq k$ and let $f$ and $g: r \rightarrow r^{-l} f(r)$ be dimension functions. Let $B \subset I^{k}$ be a Borel set and let $V$ be $a(k-l)$-dimensional linear subspace of $\mathbb{R}^{k}$. If, for a subset $S$ of $V^{\perp}$ of positive $\mathcal{H}^{l}$ measure,

$$
\mathcal{H}^{g}(B \cap(V+b))=\infty \quad \text { for all } b \in S,
$$

then $\mathcal{H}^{f}(B)=\infty$.

This will be used to prove the infinite measure case of the theorem; i.e. the next lemma.

Lemma 7. Let $m+u \leq n$. Let $\psi$ be an approximating function and let $f$ and $g: r \rightarrow r^{-(n-(m+u-1))(m-1)} f(r)$ be dimension functions with $r^{-d} f(r) \rightarrow \infty$ as $r \rightarrow 0$. Further, let $r^{-m(m+u-1)} g(r)$ be non-increasing and $r^{-(m-1)(m+u-1)} g(r)$ be increasing. If

$$
\sum_{r=1}^{\infty} f(\Psi(r)) \Psi(r)^{-(m-1) n} r^{m+u-1}=\infty
$$

then

$$
\mathcal{H}^{f}(G)=\infty
$$

Proof. Let

$$
B=\widehat{W}_{u}(m, m+u-1 ; \psi) \times I^{(n-(m+u-1))(m-1)} \subseteq I^{d} .
$$

As $\eta$ is bi-Lipschitz, from Lemma 5

$$
\mathcal{H}^{f}(G)=\mathcal{H}^{f}(\eta(B)) \asymp \mathcal{H}^{f}(B) .
$$

Since $\widehat{W}_{u}(m, m+u-1 ; \psi)$ is a limsup set, $B$ is a Borel set. Let $V$ be the space $I^{m(m+u-1)} \times\{0\}^{(m-1)(n-(m+u-1))}$ and let $S=\{0\}^{m(m+u-1)} \times I^{(n-(m+u-1))(m-1)}$. Clearly $S$ is a subset of $V^{\perp}$, and further it has positive $\mathcal{H}^{(n-(m+u-1))(m-1)}$-measure. For each $b \in S$

$$
\begin{aligned}
\mathcal{H}^{g}(B \cap(V+b)) & =\mathcal{H}^{g}\left(\left(\widehat{W}_{u}(m, m+u-1 ; \psi) \times\{0\}^{(n-(m+u-1))(m-1)}\right)+b\right) \\
& =\mathcal{H}^{g}\left(\widehat{W}_{u}(m, m+u-1 ; \psi) \times\{0\}^{(n-(m+u-1))(m-1)}\right) \\
& =\mathcal{H}^{g}\left(I^{m(m+u-1)}\right) \\
& =\infty
\end{aligned}
$$

using Theorem 2, the definition of $g$ and the fact that

$$
\sum_{r=1}^{\infty} f(\Psi(r)) \Psi(r)^{-(m-1) n} r^{m+u-1}=\infty .
$$

Thus, from Lemma $6, \mathcal{H}^{f}(G)=\infty$ as required. 
Clearly, since $G \subseteq W_{u}(m, n ; \psi)$, this implies that $\mathcal{H}^{f}\left(W_{u}(m, n ; \psi)\right)=\infty$ and completes the proof of the theorem.

\subsection{Finite measure case}

We now deal with the case where $r^{-d} f(r) \rightarrow C$ as $r \rightarrow 0$ and $C>0$ is finite. In this case $\mathcal{H}^{f}$ is comparable to $d$-dimensional Lebesgue measure and

$$
\sum_{r=1}^{\infty} f(\Psi(r)) \Psi(r)^{-(m-1) n} r^{m+u-1}=\sum_{r=1}^{\infty} \psi^{m+u-1}(r) .
$$

From Lemma 5,

$$
\left|\widehat{W}_{u}(m, m+u-1 ; \psi) \times(-1 /(m-1), 1 /(m-1))^{n-(m+u-1)(m-1)}\right|_{d} \asymp|G|_{d} .
$$

From Corollary $2,\left|W_{u}(m, m+u-1 ; \psi)\right|_{m(m+u-1)}=1$. The set of $X \in W_{u}(m$, $m+u-1 ; \psi)$ for which $\tilde{X}$ has rank at most $m-2$ has strictly smaller dimension than $\widehat{W}_{u}(m, m+u-1 ; \psi)$. Thus $\left|\widehat{W}_{u}(m, m+u-1 ; \psi)\right|_{m(m+u-1)}=1$. Hence the $d$-dimensional Lebesgue measure of $G$ is positive which further implies that the $d$ dimensional Lebesgue measure of $W_{u}(m, n ; \psi)$ is also positive and finite as required.

\section{Acknowledgments}

Our sincere thanks to Sanju Velani, Victor Beresnevich and Jason Levesley for their help, encouragement and support.

\section{References}

[1] V. I. Arnold, Geometrical Methods in the Theory of Ordinary Differential Equations (Springer-Verlag, 1983), Translated by J. Szücs.

[2] V. Beresnevich, H. Dickinson and S. L. Velani, Sets of exact 'logarithmic order' in the theory of Diophantine approximation, Math. Ann. 321(2) (2001) 253-273.

[3] _ Measure Theoretic Laws for lim sup Sets, Memoirs of the American Mathematical Society, Vol. 179, No. 846 (American Mathematical Society, Providence, RI, 2006).

[4] V. Beresnevich and S. L. Velani, Schmidt's theorem, Hausdorff measures and slicing, Int. Math. Res. Notices 2006 (2006) 1-24.

[5] - Ubiquity and a general logarithm law for geodesics, in Dynamical Systems and Diophantine Approximation, Séminaires et Congrès, Vol. 19 (Soc. Math. France, Paris, 2009), pp. 21-36.

[6] _ Classical metric Diophantine approximation revisited: The KhintchineGroshev theorem, Int. Math. Res. Notices 2010 (2010) 69-86.

[7] V. I. Bernik and M. M. Dodson, Metric Diophantine Approximation on Manifolds, Cambridge Tracts in Mathematics, Vol. 137 (Cambridge University Press, 1999).

[8] J. W. S. Cassels, An Introduction to Diophantine Approximation (Cambridge University Press, 1957).

[9] H. Dickinson, The Hausdorff dimension of systems of simultaneously small linear forms, Mathematika 40 (1993) 367-374. 
[10] — The Hausdorff dimension of sets arising in metric Diophantine approximation, Acta Arith. 68(2) (1994) 133-140.

[11] H. Dickinson and S. Velani, Hausdorff measure and linear form, J. Reine Angew. Math. 490 (1997) 1-36.

[12] M. M. Dodson, B. P. Rynne and J. A. G. Vickers, Diophantine approximation and a lower bound for Hausdorff dimension, Mathematika 37 (1990) 59-73.

[13] _ The Hausdorff dimension of exceptional sets associated with normal forms, J. London Math. Soc. (2) 49 (1994) 614-624.

[14] K. J. Falconer, Fractal Geometry - Mathematical Foundations and Applications (Wiley, 1990).

[15] A. V. Groshev, Une theoreme sur les systemes des formes lineaires, Doklady Akad. Nauk SSSR 19 (1938) 151-152.

[16] G. Harman, Metric Number Theory, London Mathematical Society Monographs, Vol. 18 (Oxford University Press, 1998).

[17] M. Hussain and J. Levesley, The metrical theory of simultaneously small linear forms, to appear in Funct. Approx. Comment. Math.

[18] V. Jarník, Über die simultanen Diophantischen appoximationen, Math. Z. 33 (1931) 505-543.

[19] A. Khinthcine, Einige Sätze über Kettenbrüche, mit anwendungen auf die theorie der Diophantischen approximationen, Math. Ann. 92 (1924) 115-125.

[20] V. Sprindžuk, Metric Theory of Diophantine Approximation (Wiley, 1979). 
Copyright of International Journal of Number Theory is the property of World Scientific Publishing Company and its content may not be copied or emailed to multiple sites or posted to a listserv without the copyright holder's express written permission. However, users may print, download, or email articles for individual use. 